\title{
ON THE SOLUTION OF SOME AXISYMMETRIC BOUNDARY VALUE PROBLEMS BY MEANS OF INTEGRAL EQUATIONS
}

\author{
VII. THE ELECTROSTATIC POTENTIAL DUE TO A SPHERICAL \\ CAP SITUATED INSIDE A CIRCULAR CYLINDER
}

\author{
by W. D. COLLINS \\ (Received 6th August, 1961)
}

\section{Introduction}

This paper is a sequel to a previous paper (1) on axisymmetric potential problems for one or more circular disks situated inside a coaxial cylinder and applies the method used for these problems to the electrostatic potential problem for a perfectly conducting thin spherical cap situated inside an earthed coaxial infinitely long circular cylinder.

We first derive in $\$ \mathbf{2}$ integral representations for the potentials of rings of point charges and dipoles and use these to construct representations for the potentials of distributions of charges and dipoles on a spherical cap, these results corresponding to those already given for a circular disk (1), (2). These representations have been used to reduce potential problems for a spherical cap to single applications of the known solution of Abel's integral equation (3), (4) and problems for two or more spherical caps to the solution of Abel and Fredholm integral equations (5), (6). We make further use of one of these representations in the electrostatic potential problem for a cap inside an earthed coaxial cylinder to show in $\S \mathbf{3}$ that this problem is governed by a Fredholm equation of the second kind. In $\S 4$ we obtain an approximate solution of this equation by iteration when the cap is maintained at a constant potential, the radius of the cap being much less than the radius of the cylinder, and give an expression for the capacity of the cap.

\section{Representations of Potential Functions for a Spherical Cap}

We first derive integral representations for the potentials of charge and dipole distributions on a spherical cap. It has been shown (1), (2) that the potential $v_{1}(\varpi, z ; \rho, \zeta)$ at any point $(\varpi, \phi, z)$ due to a ring of charges of radius $\rho$ in the plane $z=\zeta$ has the representation

$$
v_{1}(\varpi, z ; \rho, \zeta)=\frac{1}{\pi} \int_{-\rho}^{\rho} \frac{d t}{\left(\rho^{2}-t^{2}\right)^{\frac{1}{2}}\left(w^{2}+(z-\zeta+i t)^{2}\right)^{\frac{1}{2}}}, \ldots \ldots \ldots \ldots
$$

the total charge on the ring being unity. The origin $O$ for cylindrical polar 
coordinates $(\omega, \phi, z)$ is a point on the axis of the ring, this line being taken as the $z$-axis. If $(r, \theta, \phi)$ are the spherical polar coordinates of the point $(\varpi, \phi, z)$ and $\left(a, \psi, \phi^{\prime}\right)$ those of a point $\left(\rho, \phi^{\prime}, \zeta\right)$ on the ring of charges, we have

$$
\begin{aligned}
& v_{1}(r \sin \theta, r \cos \theta ; a \sin \psi, a \cos \psi) \equiv v_{1}^{\prime}(r, \theta ; a, \psi) \\
& \quad=\frac{1}{\pi} \int_{-a \sin \psi}^{a \sin \psi} \frac{d t}{\left.\left(a^{2} \sin ^{2} \psi-t^{2}\right)^{\frac{1}{2}}\left(r^{2} \sin ^{2} \theta+r \cos \theta-a \cos \psi+i t\right)^{2}\right)^{\frac{1}{2}}} .
\end{aligned}
$$

We now suitably deform the contour of integration away from the real axis into an arc of a circle of radius $a$ and make the change of variable

$$
a e^{-i \eta}=a \cos \psi-i t, \quad-\psi \leqq \eta \leqq \psi,
$$

to obtain as an alternative expression for the potential of a ring of charges

$$
v_{1}^{\prime}(r, \theta ; a, \psi)=\frac{1}{\pi} \int_{-\psi}^{\psi} \frac{d \eta}{(2 \cos \eta-2 \cos \psi)^{\frac{1}{2}}\left(r^{2} e^{i \eta}+a^{2} e^{-i \eta}-2 a r \cos \theta\right)^{\frac{1}{2}}} \ldots
$$

To obtain a representation for a ring of dipoles we consider a ring of charges of total strength $-q$ and radius $a \sin \psi$ in the plane $z=a \cos \psi$ and a ring of total strength $q$ and radius $(a+\delta a) \sin \psi$ in the plane $z=(a+\delta a) \cos \psi$, the potentials of these rings being found from (2.3). On letting $q \rightarrow \infty$ and $\delta a \rightarrow 0$ in such a way that $q \delta a \rightarrow 1$ we obtain the potential $v_{2}^{\prime}(r, \theta ; a, \psi)$ for a ring of dipoles of radius $a \sin \psi$ in the plane $z=a \cos \psi$ as

$$
v_{2}^{\prime}(r, \theta ; a, \psi)=\frac{1}{\pi} \int_{-\psi}^{\psi} \frac{\left(r \cos \theta-a e^{-i \eta}\right) d \eta}{(2 \cos \eta-2 \cos \psi)^{\frac{1}{2}}\left(r^{2} e^{i \eta}+a^{2} e^{-i \eta}-2 a r \cos \theta\right)^{\frac{3}{2}}},
$$

valid at all points other than those on the surface $r=a(0 \leqq \theta \leqq \psi)$, the total strength of the ring being unity and the axes of the dipoles being directed radially outwards from $O$. This expression we can write as

$$
\begin{aligned}
& v_{2}^{\prime}(r, \theta ; a, \psi) \\
& \quad=-\frac{1}{i \pi a} \int_{-\psi}^{\psi} \frac{\sec \frac{1}{2} \eta}{(2 \cos \eta-2 \cos \psi)^{\frac{1}{2}}} \frac{\partial}{\partial \eta}\left[\frac{\cos \frac{1}{2} \eta}{\left(r^{2} e^{i \eta}+a^{2} e^{-i \eta}-2 a r \cos \theta\right)^{\frac{1}{2}}}\right. \\
& \left.\quad+\frac{1}{2 a} \log \left[r \cos \theta-a e^{-i \eta}+\left(r^{2}+a^{2} e^{-2 i \eta}-2 a r e^{-i \eta} \cos \theta\right)^{\frac{1}{2}}\right]\right] d \eta, \ldots
\end{aligned}
$$

where the logarithm has its principal value. The representations (2.3) and (2.4) are the bricks from which we now construct representations for potentials for a spherical cap.

We take the centre $O$ and axis of the cap as origin and polar axis for spherical polar coordinates $(r, \theta, \phi)$, so that the cap, radius $a$ and semi-angle $\alpha$, is given by $r=a(0 \leqq \theta \leqq \alpha)$. The potential at a point $(r, \theta, \phi)$ of a distribution of rings of charges on the cap, the density of the distribution being $\sigma(\psi)$ per unit area at the point $\left(a, \psi, \phi^{\prime}\right)$, is

$$
V(r, \theta)=\int_{0}^{\alpha} 2 \pi a^{2} \sin \psi \sigma(\psi) v_{1}^{\prime}(r, \theta ; a, \psi) d \psi
$$


from which, by using (2.3) and interchanging the order of integration, we obtain

$$
V(r, \theta)=\frac{a}{2} \int_{-\alpha}^{\alpha} \frac{g(\eta) d \eta}{\left(r^{2} e^{i \eta}+a^{2} e^{-i \eta}-2 a r \cos \theta\right)^{\frac{1}{2}}},
$$

where $g(\eta)$ is a real even function given by

$$
g(\eta)=4 a \int_{\eta}^{a} \frac{\sigma(\psi) \sin \psi d \psi}{(2 \cos \eta-2 \cos \psi)^{\frac{1}{2}}} \quad(\eta \geqq 0) .
$$

Similarly, the potential at the point $(r, \theta, \phi)$ of a distribution of rings of dipoles on the cap, the density of the distribution being $\tau(\psi)$ per unit area at the point $\left(a, \psi, \phi^{\prime}\right)$ and the axes of the dipoles being directed radially outwards from the centre of the cap, is

$$
V(r, \theta)=\int_{0}^{a} 2 \pi a^{2} \sin \psi \tau(\psi) v_{2}^{\prime}(r, \theta ; a, \psi) d \psi .
$$

Using (2.4), interchanging the order of integration and carrying out an integration by parts, we obtain

$$
\begin{aligned}
V(r, \theta)= & \frac{1}{2 i} \int_{-\alpha}^{\alpha} j(\eta)\left[\frac{2 \cos \frac{1}{2} \eta}{\left(r^{2} e^{i \eta}+a^{2} e^{-i \eta}-2 a r \cos \theta\right)^{\frac{1}{2}}}\right. \\
& \left.+\frac{1}{a} \log \left[r \cos \theta-a e^{-i \eta}+\left(r^{2}+a^{2} e^{-2 i \eta}-2 a r e^{-i \eta} \cos \theta\right)^{\frac{1}{2}}\right]\right] d \eta, \ldots \ldots .
\end{aligned}
$$

where $j(\eta)$ is a real odd function given by

$$
j(\eta)=2 a \frac{d}{d \eta}\left[\sec \frac{1}{2} \eta \int_{\eta}^{a} \frac{\tau(\psi) \sin \psi d \psi}{(2 \cos \eta-2 \cos \psi)^{\frac{1}{2}}}\right] \quad(\eta>0) .
$$

This expression for $V(r, \theta)$ is valid at all points including those on the cap (3). The stream-function corresponding to the potential (2.6) is

$$
\begin{aligned}
\Psi(r, \theta)=\frac{1}{2 i} \int_{-\alpha}^{\alpha} j(\eta) & {\left[\frac{r e^{\frac{1}{2 i \eta}} \cos \theta-a e^{-\frac{1}{2} i \eta}}{\left(r^{2} e^{i \eta}+a^{2} e^{-i \eta}-2 a r \cos \theta\right)^{\frac{1}{2}}}\right.} \\
& \left.-\frac{r\left(a e^{-\frac{1}{2} i \eta} \cos \theta-r e^{+i \eta}\right)}{a\left(r^{2} e^{i \eta}+a^{2} e^{-i \eta}-2 a r \cos \theta\right)^{\frac{1}{2}}}\right] d \eta
\end{aligned}
$$
that

The square roots in these various expressions have positive real parts, so

$$
\begin{aligned}
\left(r^{2} e^{i \eta}+a^{2} e^{-i \eta}-2 a r \cos \theta\right)^{\frac{1}{2}} & =\rho e^{\frac{1}{2} i \tau} \text { for } r \geqq a, \\
& =\rho e^{-\frac{1}{2} i \tau} \text { for } \quad r<a,
\end{aligned}
$$

with $\rho \geqq 0, \quad 0 \leqq \tau \leqq \pi$ for $\quad 0 \leqq \eta \leqq \alpha$,

$$
-\pi<\tau \leqq 0 \text { for }-\alpha \leqq \eta<0 \text {. }
$$


When $r=a$, we have

$$
\begin{aligned}
\left(r^{2} e^{i \eta}+a^{2} e^{-i \eta}-2 a r \cos \theta\right)^{\frac{1}{2}} & =a(2 \cos \eta-2 \cos \theta)^{\frac{1}{2}} & & (\theta \geqq|\eta|), \\
& = \pm i a(2 \cos \theta-2 \cos \eta)^{\frac{1}{2}} & & (\theta<|\eta|)
\end{aligned}
$$

if $r \rightarrow a$ through values greater than $a$,

$$
=\mp i a(2 \cos \theta-2 \cos \eta)^{\frac{1}{2}} \quad(\theta<|\eta|)
$$

if $r \rightarrow a$ through values less than $a$,

the upper signs holding for $0 \leqq \eta \leqq \alpha$ and the lower for $-\alpha \leqq \eta<0$. The integral (2.5) is interpreted as a Cauchy integral at the point $(r=a, \theta=0)$.

The potential at any point when the cap is maintained at a given axisymmetric potential is due to a distribution of charges induced on the cap, and hence as is shown in a previous paper (3) we represent this potential by (2.5), determining $g(\eta)$ by one application of the known solution of Abel's integral equation. Similarly, for a flow of perfect fluid past the cap the potential at any point is due to a distribution of doublets or dipoles induced on the cap, the axes of the doublets being normal to the cap, and we represent this potential (4) by (2.6) and the corresponding stream-function (3) by (2.7).

These representations can also be used to investigate potential problems for a spherical cap situated inside a coaxial circular cylinder, and we now illustrate this by considering the electrostatic problem for this configuration.

\section{The Electrostatic Potential due to a Spherical Cap inside a Circular Cylinder}

We consider a spherical cap maintained at a constant potential in an external electrostatic field and situated inside an earthed coaxial infinitely long hollow cylinder. We use spherical polar coordinates $(r, \theta, \phi)$ and cylindrical polar coordinates $(\varpi, \phi, z)$ with the centre and axis of the cap as origin and polar axis, so that the cap, radius $a$ and semi-angle $\alpha$, is given by $r=a(0 \leqq \theta \leqq \alpha)$ and the cylinder, radius $c$, by $\varpi=c(-\infty<z<\infty)$. Since the cap lies wholly within the cylinder, we have $c>\max (a \sin \theta)$ for $0 \leqq \theta \leqq \alpha$. If $V_{1}(\varpi, z)$ is the potential due to the external electrostatic field in infinite space, the singularities of the field lying in the region $0 \leqq \varpi<c(-\infty<z<\infty)$ but not on the surface $r=a(0 \leqq \theta \leqq \alpha)$, the total potential $V(w, z)$ due to the cap, maintained at a constant potential $V_{0}$ in this field and situated inside the earthed cylinder, is

$$
V(\varpi, z)=V_{1}(\varpi, z)+U(r, \theta),
$$

where $U(r, \theta)$ is a potential satisfying the conditions

$$
\begin{aligned}
& U(r, \theta)=f(\theta)=V_{0}-V_{1}(a \sin \theta, a \cos \theta) \\
& \quad \text { on the cap } r=a(0 \leqq \theta \leqq \alpha), \\
& =j(z)=-V_{1}(c, z) \text { on the cylinder } \varpi=c(-\infty<z<\infty) .
\end{aligned}
$$

Further, $U(r, \theta)$ is continuously differentiable at all interior points of the cylinder except those on the cap, whilst $U$ and $\partial U / \partial r$ are continuous for approach 
to the cap except that for points on the rim $(r=a, \theta=\alpha)$ of the cap $\partial U / \partial r$ tends to infinity as the inverse square root of the distance from the edge: Finally, $U(r, \theta)$ is $0\left(|z|^{-1}\right)$ for large $|z|$.

This potential $U(r, \theta)$ is due to distributions of charges induced on the cap and the cylinder, and hence we represent it as

$$
\begin{aligned}
U(r, \theta)=\frac{a}{2} \int_{-\alpha}^{a} \frac{g(\eta) d \eta}{\left(r^{2} e^{i \eta}+a^{2} e^{-i \eta}-2 a r \cos \theta\right)^{\frac{1}{2}}} \\
+\int_{0}^{\infty}\left(h_{1}(\lambda) \cos \lambda z+h_{2}(\lambda) \sin \lambda z\right) I_{0}(\lambda \sigma) d \lambda,
\end{aligned}
$$

where $g(\eta), h_{1}(\lambda)$, and $h_{2}(\lambda)$ are real continuous functions, $g(\eta)$ being even, and $I_{0}(\lambda \varpi)$ is the modified Bessel function of the first kind of order zero (7; p. 77). The first integral has the form (2.5) and is the potential of a distribution of charges on the cap, whilst the second integral is a solution of Laplace's equation appropriate to the interior of the cylinder and is a representation of the potential due to a distribution of charges on the cylinder. The potential (3.4) can be shown to satisfy the conditions of the problem other than (3.2) and (3.3) by the methods used in a previous paper (3) for the potential for a cap in infinite space. We now use conditions (3.2) and (3.3) on the cap and the cylinder to derive a Fredholm equation governing the problem.

We first consider the condition (3.2) satisfied by $U(r, \theta)$ on the cap $r=a(0 \leqq \theta \leqq \alpha)$. It can be shown (3) that the limit of the first integral in (3.4) as the point $(r, \theta, \phi)$ approaches a point on the cap is the value of the integral at that point, and hence from (2.8) and (2.9) we have that

$$
\begin{array}{r}
\int_{0}^{\theta} \frac{g(\eta) d \eta}{(2 \cos \eta-2 \cos \theta)^{\frac{1}{2}}}+\int_{0}^{\infty}\left(h_{1}(\lambda) \cos (\lambda a \cos \theta)+h_{2}(\lambda) \sin (\lambda a \cos \theta)\right) \\
\quad \times I_{0}(\lambda a \sin \theta) d \lambda=f(\theta) \quad(0 \leqq \theta \leqq \alpha) . \ldots . . .
\end{array}
$$

This we regard as an Abel integral equation for $g(\eta)$, the first integral in (3.5) reducing to the usual form of this equation after a change of variable, and on solving it obtain $g(\eta)$ in terms of $h_{1}(\lambda)$ and $h_{2}(\lambda)$. To do this we need to express the second integral in (3.5) in the same form as the first.

Since $I_{0}(\lambda \omega) \cos \lambda z$ and $I_{0}(\lambda \omega) \sin \lambda z$ are symmetrical potentials which have the finite values $\cos \lambda z$ and $\sin \lambda z$ on the axis $\pi=0$, we use Laplace's expression (8) for such potentials to obtain

$$
\begin{aligned}
I_{0}(\lambda \varpi) \exp (i \lambda z) & =\frac{1}{\pi} \int_{0}^{\pi} \exp [i \lambda(z+i \varpi \cos \chi)] d \chi \\
& =\frac{1}{\pi} \int_{-\infty}^{\infty} \frac{\exp [i \lambda(z+i t)] d t}{\left(\varpi^{2}-t^{2}\right)^{\frac{1}{2}}} .
\end{aligned}
$$

When $z=a \cos \theta$ and $\pi=a \sin \theta$, we have

$$
\begin{aligned}
& I_{0}(\lambda a \sin \theta) \exp (i \lambda a \cos \theta)=\frac{1}{\pi} \int_{-a \sin \theta}^{a \sin \theta} \frac{\exp [i \lambda(a \cos \theta+i t)] d t}{\left(a^{2} \sin ^{2} \theta-t^{2}\right)^{t}} . \\
& \text { E.M.S. }-\mathrm{B}
\end{aligned}
$$


If we now suitably deform the contour of integration away from the real axis into an arc of a circle of radius $a$ and make the change of variable

$$
a e^{i \eta}=a \cos \theta+i t, \quad-\theta \leqq \eta \leqq \theta,
$$

we obtain

$$
I_{0}(\lambda a \sin \theta) \exp (i \lambda a \cos \theta)=\frac{1}{\pi} \int_{-\theta}^{\theta} \frac{\exp \left[i\left(\frac{1}{2} \eta+\lambda a e^{i \eta}\right)\right] d \eta}{(2 \cos \eta-2 \cos \theta)^{\frac{1}{2}}} .
$$

Taking the real and imaginary parts of this expression, we have

and

$$
I_{0}(\lambda a \sin \theta) \cos (\lambda a \cos \theta)=\frac{2}{\pi} \int_{0}^{\theta} \frac{M_{1}(\eta, \lambda) d \eta}{(2 \cos \eta-2 \cos \theta)^{\frac{1}{2}}}
$$

where

$$
I_{0}(\lambda a \sin \theta) \sin (\lambda a \cos \theta)=\frac{2}{\pi} \int_{0}^{\theta} \frac{M_{2}(\eta, \lambda) d \eta}{(2 \cos \eta-2 \cos \theta)^{\frac{1}{2}}}
$$

$M_{1}(\eta, \lambda)=\cos \frac{1}{2} \eta \cos (\lambda a \cos \eta) \cosh (\lambda a \sin \eta)$

and

$$
+\sin \frac{1}{2} \eta \sin (\lambda a \cos \eta) \sinh (\lambda a \sin \eta) \text {. }
$$

$M_{2}(\eta, \lambda)=\cos \frac{1}{2} \eta \sin (\lambda a \cos \eta) \cosh (\lambda a \sin \eta)$

$$
-\sin \frac{1}{2} \eta \cos (\lambda a \cos \eta) \sinh (\lambda a \sin \eta)
$$

Using these results, we write the second integral in (3.5) in the same form as the first and hence obtain

where

$$
\int_{0}^{\theta} \frac{F(\eta) d \eta}{(2 \cos \eta-2 \cos \theta)^{\frac{1}{2}}}=f(\theta) \quad(0 \leqq \theta \leqq \alpha),
$$

$$
g(\eta)+\frac{2}{\pi} \int_{0}^{\infty}\left(h_{1}(\lambda) M_{1}(\eta, \lambda)+h_{2}(\lambda) M_{2}(\eta, \lambda)\right) d \lambda=F(\eta) \quad(0 \leqq \eta \leqq \alpha) \ldots
$$

Equation (3.8) reduces to Abel's integral equation after a change of variable, and its solution is found as

$$
F(\eta)=\frac{2}{\pi} \frac{d}{d \eta} \int_{0}^{\eta} \frac{f(\theta) \sin \theta d \theta}{(2 \cos \theta-2 \cos \eta)^{\frac{1}{2}}} \quad(0 \leqq \eta \leqq \alpha) ;
$$

hence $F(\eta)$ is known and further is even.

The condition (3.3) on the cylinder $m=c(-\infty<z<\infty)$ gives

$$
\begin{aligned}
\int_{0}^{\infty}\left(h_{1}(\lambda) \cos \lambda z\right. & \left.+h_{2}(\lambda) \sin \lambda z\right) I_{0}(\lambda c) d \lambda \\
& +\frac{a}{2} \int_{-\alpha}^{\alpha} \frac{e^{-\frac{1}{2} i \eta} g(\eta) d \eta}{\left(c^{2}+\left(z-a e^{-i \eta}\right)^{2}\right)^{\frac{1}{2}}}=j(z) \quad(-\infty<z<\infty) .
\end{aligned}
$$

On replacing $z$ by $-z$ in this equation we obtain a second equation for $h_{1}(\lambda)$ 
and $h_{2}(\lambda)$ as

$$
\begin{aligned}
\int_{0}^{\infty}\left(h_{1}(\lambda) \cos \lambda z-h_{2}(\lambda) \sin \lambda z\right) I_{0}(\lambda c) d \lambda \\
+\frac{a}{2} \int_{-\alpha}^{\alpha} \frac{e^{-\frac{1}{2} i \eta} g(\eta) d \eta}{\left(c^{2}+\left(z+a e^{-i \eta}\right)^{2}\right)^{\frac{1}{2}}}=j(-z) \quad(-\infty<z<\infty) .
\end{aligned}
$$

On adding and subtracting (3.11) and (3.12) we obtain equations determining $h_{1}(\lambda)$ and $h_{2}(\lambda)$, these being

$2 \int_{0}^{\infty} h_{1}(\lambda) I_{0}(\lambda c) \cos \lambda z d \lambda$

$$
+\frac{a}{2} \int_{-\alpha}^{\alpha} e^{-\frac{1}{2} i \eta} g(\eta)\left[\frac{1}{\left(c^{2}+\left(z-a e^{-i \eta}\right)^{2}\right)^{\frac{1}{2}}}+\frac{1}{\left(c^{2}+\left(z+a e^{-i \eta}\right)^{2}\right)^{\frac{1}{2}}}\right] d \eta=2 j_{+}(z),
$$

and

$$
(-\infty<z<\infty) \text {. }
$$

$$
\begin{aligned}
& 2 \int_{0}^{\infty} h_{2}(\lambda) I_{0}(\lambda c) \sin \lambda z d \lambda \\
& \quad+\frac{a}{2} \int_{-\alpha}^{\alpha} e^{-\frac{1}{2} i \eta} g(\eta)\left[\frac{1}{\left(c^{2}+\left(z-a e^{-i \eta}\right)^{2}\right)^{\frac{1}{2}}}-\frac{1}{\left(c^{2}+\left(z+a e^{-i \eta}\right)^{2}\right)^{\frac{1}{2}}}\right] d \eta=2 j_{-}(z),
\end{aligned}
$$

where

$$
(-\infty<z<\infty)
$$

$$
j_{ \pm}(z)=\frac{1}{2}(j(z) \pm j(-z)) .
$$

We now invert equations (3.13) and (3.14) to find $h_{1}(\lambda)$ and $h_{2}(\lambda)$ as $I_{0}(\hat{\lambda} c) h_{1}(\lambda)=J_{+}(\lambda)$

$$
\begin{array}{r}
-\frac{a}{2 \pi} \int_{0}^{\infty} \cos \lambda z \int_{-\alpha}^{\alpha} e^{-\frac{1}{2} i \eta} g(\eta)\left[\frac{1}{\left(c^{2}+\left(z-a e^{-i \eta}\right)^{2}\right)^{\frac{1}{2}}}\right. \\
\left.+\frac{1}{\left(c^{2}+\left(z+a e^{-i \eta}\right)^{2}\right)^{\frac{1}{2}}}\right] d \eta d z
\end{array}
$$

and

$I_{0}(\lambda c) h_{2}(\lambda)=J_{-}(\lambda)$

$$
\begin{array}{r}
-\frac{a}{2 \pi} \int_{0}^{\infty} \sin \lambda z \int_{-\alpha}^{\alpha} e^{-\frac{1}{2} i \eta} g(\eta)\left[\frac{1}{\left(c^{2}+\left(z-a e^{-i \eta}\right)^{2}\right)^{\frac{1}{2}}}\right. \\
\left.-\frac{1}{\left(c^{2}+\left(z+a e^{-i \eta}\right)^{2}\right)^{\frac{3}{2}}}\right] d \eta d z
\end{array}
$$

where

$$
J_{+}(\lambda)=\frac{2}{\pi} \int_{0}^{\infty} j_{+}(z) \cos \lambda z d z, \quad J_{-}(\lambda)=\frac{2}{\pi} \int_{0}^{\infty} j_{-}(z) \sin \lambda z d z .
$$

If in (3.15) we interchange the order of integration in the integral on the 
right-hand side and note that $g(\eta)$ is even, we find we need to evaluate the integral

$$
\begin{aligned}
I(\eta, \lambda)= & \int_{0}^{\infty}\left[\frac{e^{-\frac{1}{2} i \eta}}{\left(c^{2}+\left(z-a e^{-i \eta}\right)^{2}\right)^{\frac{1}{2}}}+\frac{e^{\frac{1}{2} i \eta}}{\left(c^{2}+\left(z-a e^{i \eta}\right)^{2}\right)^{\frac{1}{2}}}\right. \\
& \left.+\frac{e^{-\frac{1}{2} i \eta}}{\left(c^{2}+\left(z+a e^{-i \eta}\right)^{2}\right)^{\frac{1}{2}}}+\frac{e^{\frac{1}{2} i \eta}}{\left(c^{2}+\left(z+a e^{i \eta}\right)^{2}\right)^{\frac{1}{2}}}\right] \cos \lambda z d z .
\end{aligned}
$$

By considering the integrals (7, p. 410$)$

$$
\frac{2}{\pi} \int_{0}^{\infty} K_{0}(\lambda c) \cos \left[\lambda\left(z \pm a e^{-i \eta}\right)\right] d \lambda=\frac{1}{\left(c^{2}+\left(z \pm a e^{-i \eta}\right)^{2}\right)^{\frac{1}{2}}},
$$

where $a|\sin \eta|<c$ and $K_{0}(\lambda c)$ is the modified Bessel function of the third kind of order zero $(7$, p. 78$)$, we can show that

$$
I(\eta, \lambda)=4 K_{0}(\lambda c) M_{1}(\eta, \lambda)
$$

where $M_{1}(\eta, \lambda)$ is given by (3.6), provided $a|\sin \eta|<c$. This last condition is satisfied for all $\eta$ since the cap lies wholly within the cylinder. Equation (3.15) thus gives

$$
\left.I_{0}(\lambda c) h_{1}(\lambda)=J_{+}, \lambda\right)-\frac{2 a K_{0}(\lambda c)}{\pi} \int_{0}^{a} g(\xi) M_{1}(\xi, \lambda) d \xi \quad(0 \leqq \lambda<\infty) \ldots
$$

Similarly, from (3.16) we find that

$$
I_{0}(\lambda c) h_{2}(\lambda)=J_{-}(\lambda)-\frac{2 a K_{0}(\lambda c)}{\pi} \int_{0}^{\alpha} g(\xi) M_{2}(\xi, \lambda) d \xi, \quad(0 \leqq \lambda<\infty), \ldots(3
$$

where $M_{2}(\xi, \lambda)$ is given by (3.7).

We next substitute these expressions for $h_{1}(\lambda)$ and $h_{2}(\lambda)$ in (3.9) to obtain

where

$$
g(\eta)-\frac{1}{\pi} \int_{0}^{\alpha} K(\xi, \eta) g(\xi) d \xi=G(\eta), \quad(0 \leqq \eta \leqq \alpha)
$$

and

$$
K(\xi, \eta)=\frac{4 a}{\pi} \int_{0}^{\infty} \frac{K_{0}(\lambda c)}{I_{0}(\lambda c)}\left[M_{1}(\xi, \lambda) M_{1}(\eta, \lambda)+M_{2}(\xi, \lambda) M_{2}(\eta, \lambda)\right] d \lambda
$$

$$
G(\eta)=F(\eta)-\frac{2}{\pi} \int_{0}^{\infty} \frac{\left[J_{+}(\lambda) M_{1}(\eta, \lambda)+J_{-}(\lambda) M_{2}(\eta, \lambda)\right]}{I_{0}(\lambda c)} d \lambda . \ldots
$$

Since $G(\eta)$ is known, this is a Fredholm integral equation determining $g(\eta)$. We can however show that

where

$$
K(\xi, \eta)=k(\xi, \eta)+k(-\xi, \eta)
$$

$k(\xi, \eta)$

$$
\begin{aligned}
& =\frac{2 a}{\pi} \int_{0}^{\infty} \frac{K_{0}(\lambda c)}{I_{0}(\lambda c)}\left[\cos [\lambda a(\cos \xi-\cos \eta)] \cosh [\lambda a(\sin \xi+\sin \eta)] \cos \frac{1}{2}(\xi-\eta)\right. \\
& \left.\quad+\sin [\lambda a(\cos \xi-\cos \eta)] \sinh [\lambda a(\sin \xi+\sin \eta)] \sin \frac{1}{2}(\xi-\eta)\right] d \lambda, \ldots(3.21)
\end{aligned}
$$


so that we replace (3.19) by the equation

$$
g(\eta)-\frac{1}{\pi} \int_{-\alpha}^{\alpha} k(\xi, \eta) g(\xi) d \xi=G(\eta), \quad(-\alpha \leqq \eta \leqq \alpha)
$$

$g(\eta)$ and $G(\eta)$ being even functions, and take this as the integral equation determining $g(\eta)$. It may be noted that this equation has a symmetric kernel. The functions $h_{1}(\lambda)$ and $h_{2}(\lambda)$ are then given in terms of $g(\eta)$ by (3.17) and (3.18).

An exact solution of (3.22) is not known, and for general values of $a$ and $c$ it is necessary to solve it numerically. When the ratio $a / c$ is sufficiently small however, an iterative solution can be obtained as a convergent power series, the first few terms of which provide a good approximation to $g(\eta)$. To obtain this approximate solution we expand $k(\xi, \eta)$ in powers of $a / c$ to give

$$
\begin{aligned}
& \begin{array}{r}
k(\xi, \eta)=\frac{a}{\pi c} \int_{0}^{\infty} \frac{K_{0}(u)}{I_{0}(u)}\left[\exp \left[\frac{i}{2}(\xi-\eta)\right] \cos \left[\frac{u a}{c}\left(e^{i \xi}-e^{-i \eta}\right)\right]\right. \\
\left.+\exp \left[-\frac{i}{2}(\xi-\eta)\right] \cos \left[\frac{u a}{c}\left(e^{-i \xi}-e^{i \eta}\right)\right]\right] d u
\end{array} \\
& \text { where }=\frac{1}{\pi} \sum_{n=0}^{\infty} \frac{(n !)^{2}}{(2 n+1) !} \beta_{2 n}\left(\frac{2 a}{c}\right)^{2 n+1} \times \cos \left[\left(n+\frac{1}{2}\right)(\xi-\eta)\right] \sin ^{2 n \frac{1}{2}(\xi+\eta), \ldots} \\
& \qquad \beta_{2 n}=\frac{(2 n+1)}{(n !)^{2}} \int_{0}^{\infty} \frac{u^{2 n} K_{0}(u)}{I_{0}(u)} d u
\end{aligned}
$$

where$$
=\frac{1}{(n !)^{2}} \int_{0}^{\infty} \frac{u^{2 n} d u}{I_{0}^{2}(u)} \text {. }
$$

The coefficients $\beta_{2 n}$ are tabulated by Smythe (9) for integer values of $n$ from 0 to 41 , the first three coefficients being

$$
\beta_{0}=1 \cdot 3677, \quad \beta_{2}=1.9407, \beta_{4}=2 \cdot 5873 .
$$

\section{The Cap Maintained at a Constant Potential}

When the cap is maintained at a constant potential $V_{0}$ in the absence of an external field, we have

so that

$$
f(\theta)=V_{0}, j(z)=0,
$$

$$
J_{+}(\lambda)=J_{-}(\lambda)=0
$$

and from equations (3.10) and (3.20) we obtain

$$
G(\eta)=F(\eta)=\frac{2 V_{0}}{\pi} \cos \frac{1}{2} \eta
$$

Thus the equation to be solved for $g(\eta)$ is

$$
g(\eta)-\frac{1}{\pi} \int_{-\alpha}^{\alpha} k(\xi, \eta) g(\xi) d \xi=\frac{2 V_{0}}{\pi} \cos \frac{1}{2} \eta \quad(-\alpha \leqq \eta \leqq \alpha) .
$$


The capacity $C$ of the cap is then given by

$$
V_{0} C=a \int_{0}^{a} g(\eta) \cos \frac{1}{2} \eta d \eta
$$

Using (3.23) and writing $\sigma=a / c$, we find the approximate solution of (4.1) by iteration as

$$
\begin{aligned}
& g(\eta)=\frac{2 V_{0}}{\pi}\left[\cos \frac{1}{2} \eta+\frac{2 \beta_{0} \sigma}{\pi^{2}}(\alpha+\sin \alpha) \cos \frac{1}{2} \eta+\frac{4 \beta_{0}^{2} \sigma^{2}}{\pi^{4}}(\alpha+\sin \alpha)^{2} \cos \frac{1}{2} \eta\right. \\
& +\frac{\sigma^{3}}{\pi^{2}}\left[-\frac{\beta_{2}}{3}\left(\frac{1}{6}(3 \sin 2 \alpha+2 \sin 3 \alpha) \cos \frac{1}{2} \eta-(2 \sin \alpha+\sin 2 \alpha) \cos \frac{3}{2} \eta\right.\right. \\
& \left.\left.+(\alpha+\sin \alpha) \cos \frac{5}{2} \eta\right)+\frac{8 \beta_{0}^{3}}{\pi^{4}}(\alpha+\sin \alpha)^{3} \cos \frac{1}{2} \eta\right] \\
& +\frac{\sigma^{4}}{\pi^{4}}\left[\frac { \beta _ { 0 } \beta _ { 2 } } { 3 } \left(\left[(2 \sin \alpha+\sin 2 \alpha)^{2}-(\alpha+\sin \alpha)(3 \sin 2 \alpha+2 \sin 3 \alpha)\right] \cos \frac{1}{2} \eta\right.\right. \\
& \left.+2(\alpha+\sin \alpha)(2 \sin \alpha+\sin 2 \alpha) \cos \frac{3}{2} \eta-2(\alpha+\sin \alpha)^{2} \cos \frac{5}{2} \eta\right) \\
& \left.+\frac{16 \beta_{0}^{4}}{\pi^{4}}(\alpha+\sin \alpha)^{4} \cos \frac{1}{2} \eta\right] \\
& +\frac{\sigma^{5}}{\pi^{2}}\left[\frac { \beta _ { 4 } } { 1 5 } \left(\frac{1}{20}(5 \sin 4 \alpha+4 \sin 5 \alpha) \cos \frac{1}{2} \eta-\frac{1}{3}(4 \sin 3 \alpha+3 \sin 4 \alpha) \cos \frac{3}{2} \eta\right.\right. \\
& \left.+(3 \sin 2 \alpha+2 \sin 3 \alpha) \cos \frac{5}{2} \eta-2(2 \sin \alpha+\sin 2 \alpha) \cos \frac{7}{2} \eta+(\alpha+\sin \alpha) \cos \frac{9}{2} \eta\right) \\
& +\frac{2 \beta_{0}^{2} \beta_{2}}{3 \pi^{4}}(\alpha+\sin \alpha)\left(\frac { 1 } { 3 } \left[6(2 \sin \alpha+\sin 2 \alpha)^{2}\right.\right. \\
& -5(\alpha+\sin \alpha)(3 \sin 2 \alpha+2 \sin 3 \alpha)] \cos \frac{1}{2} \eta \\
& \left.+2(\alpha+\sin \alpha)(2 \sin \alpha+\sin 2 \alpha) \cos \frac{3}{2} \eta-2(\alpha+\sin \alpha)^{2} \cos \frac{5}{2} \eta\right) \\
& \left.\left.+\frac{32 \beta_{0}^{5}}{\pi^{8}}(\alpha+\sin \alpha)^{5} \cos \frac{1}{2} \eta\right]\right]+O\left(\sigma^{6}\right) .
\end{aligned}
$$

The capacity of the cap is then found from (4.2) as

$$
\begin{aligned}
\frac{C}{a} & =\frac{(\alpha+\sin \alpha)}{\pi}+\frac{2 \beta_{0} \sigma}{\pi^{3}}(\alpha+\sin \alpha)^{2}+\frac{4 \beta_{0}^{2} \sigma^{2}}{\pi^{5}}(\alpha+\sin \alpha)^{3} \\
& +\frac{\sigma^{3}}{\pi^{3}}\left(\frac{\beta_{2}}{6} P(\alpha)+\frac{8 \beta_{0}^{3}}{\pi^{4}}(\alpha+\sin \alpha)^{4}\right) \\
& +\frac{\sigma^{4}}{\pi^{5}}(\alpha+\sin \alpha)\left(\frac{2 \beta_{0} \beta_{2}}{3} P(\alpha)+\frac{16 \beta_{0}^{4}}{\pi^{4}}(\alpha+\sin \alpha)^{4}\right) \\
& +\frac{\sigma^{5}}{\pi^{3}}\left(\frac{\beta_{4}}{15} Q(\alpha)+\frac{2 \beta_{0}^{2} \beta_{2}}{\pi^{4}}(\alpha+\sin \alpha)^{2} P(\alpha)+\frac{32 \beta_{0}^{5}}{\pi^{8}}(\alpha+\sin \alpha)^{6}\right)+O\left(\sigma^{6}\right),
\end{aligned}
$$


where

and

$$
P(\alpha)=(2 \sin \alpha+\sin 2 \alpha)^{2}-\frac{2}{3}(\alpha+\sin \alpha)(3 \sin 2 \alpha+2 \sin 3 \alpha)
$$

$$
\begin{aligned}
Q(\alpha) & =\frac{1}{10}(\alpha+\sin \alpha)(5 \sin 4 \alpha+4 \sin 5 \alpha) \\
& -\frac{1}{3}(2 \sin \alpha+\sin 2 \alpha)(4 \sin 3 \alpha+3 \sin 4 \alpha) \\
& +\frac{1}{6}(3 \sin 2 \alpha+2 \sin 3 \alpha)^{2} .
\end{aligned}
$$

When $\alpha$ equals $\pi$, the cap becomes a sphere and we have that

$$
\begin{aligned}
\frac{C}{a} & =1+\frac{2 \beta_{0}}{\pi} \sigma+\frac{4 \beta_{0}^{2}}{\pi^{2}} \sigma^{2}+\frac{8 \beta_{0}^{3}}{\pi^{3}} \sigma^{3}+\frac{16 \beta_{0}^{4}}{\pi^{4}} \sigma^{4}+\frac{32 \beta_{0}^{5}}{\pi^{5}} \sigma^{5}+O\left(\sigma^{6}\right) \\
& =1+0.8707 \sigma+0.7581 \sigma^{2}+0.6601 \sigma^{3}+0.5747 \sigma^{4}+0.5004 \sigma^{5}+O\left(\sigma^{6}\right) .
\end{aligned}
$$

The problem of a sphere situated inside a circular cylinder has previously been investigated by Smythe (9) and Knight (10) using other methods.

When $\alpha$ tends to zero and $a$ tends to infinity in such a way that $a \alpha$ tends to a finite limit $b$, the cap becomes a disk of radius $b$. The limit of (4.3) then gives the expression for the capacity of a disk situated inside a cylinder obtained previously (1).

Finally, the problems of the flow of perfect fluid past a spherical cap in a cylinder and of the slow steady rotation of a cap in a cylinder containing viscous fluid can be investigated using the method of this paper and the representations (2.6) and (2.7).

\section{REFERENCES}

(1) W. D. Collins, To be published.

(2) W. D. Collins, Quart. J. Mech. App. Math., 14 (1961), 101-117.

(3) W. D. Collins, Quart. J. Mech. App. Math., 12 (1959), 232-241.

(4) W. D. Collins, Mathematika, 6 (1959), 120-133.

(5) W. D. Collins, Proc. London Math. Soc., (3) 10 (1960), 428-460.

(6) W. D. Collins, Proc. Edin. Math. Soc., (2) 12 (1960), 95-106. 1944).

(7) G. N. Watson, Theory of Bessel Functions (Second Edition, Cambridge,

(8) H. Bateman, Partial Differential Equations of Mathematical Physics (Cambridge, 1932), 405-408.

(9) W. R. SмYthe, J. App. Physics, 31 (1960), 553-556.

(10) R. C. KNIGHT, Quart. J. Math., 7 (1936), 124-133.

\section{Department OF MATHEMATICS}

THE UNIVERSITY

MANCHESTER 